\title{
Enhancing Wake-Up Radio Range Through Minimum Energy Coding
}

\author{
Nour El Hoda Djidi, Matthieu Gautier, Antoine Courtay and Olivier Berder \\ Univ. Rennes, CNRS, IRISA, France \\ nour-el-hoda.djidi@irisa.fr, matthieu.gautier@irisa.fr, antoine.courtay@irisa.fr, olivier.berder@irisa.fr
}

\begin{abstract}
A substantial part of the research on wireless sensor networks is focused on the optimization of the energy consumption through either hardware or protocol communication stacks. Wake-up Receivers (WuRs) represent a new paradigm that offers both ultra low power consumption and low latency through asynchronous communications. However, WuRs have a low sensitivity and thus can misinterpret the received signal inducing a performance degradation of the whole communicating system. To tackle this issue, low power channel coding techniques can be used and we propose in this work to apply Hamming coding and Minimum Energy Coding (ME) to enhance WuR range. A performance study of these two types of coding shows that ME coding outperforms Hamming code in reducing both bit error rate and energy consumption. At a range of $28 \mathrm{~m}$, ME coding saves about 3 times the energy at a bit error rate of $10^{-3}$ compared to uncoded scheme. Furthermore, experimentation on the missed wake-ups when applying ME coding was done, showing a gain of $22 \%$ in reliability compared to uncoded scheme.
\end{abstract}

Index Terms - Internet of things, Energy efficiency, Minimum energy coding, Wake-up receivers, Wireless sensor networks

\section{INTRODUCTION}

Internet of things are emerging in our world with huge deployments for different applications such as smart cities, smart manufactures, and smart health [1]. Generally the things form a Wireless Sensor Network (WSN) with sensing and actuating capabilities [2]. The nodes in WSNs are battery powered and have a limited lifetime. Thus, keeping nodes alive as long as possible is one of the most important concerns of researchers in this field [3].

One approach to save energy is to use duty cycled protocols. This technique consists in periodically activating nodes according to a duty cycle. Unfortunately it still suffers from idle listening and overhearing inducing a waste of energy and a long latency [4]. A more recent approach is the use of wakeup receivers (WuRs) [5].

WuR consists of an ultra low power secondary radio consuming a few microwatts, whereas the main radio consumes in the order of milliwatts when being activated. WuR is always listening to the channel, while the main node is deactivated. It serves as a tool to wake up the latter with low latency, when a specific signal, called Wake-up Beacon (WUB), is received, containing the address of the intended node. Most of WuRs have computing capabilities performed by an Ultra-Low Power

This work is part of the project Wake-Up funded by the French National Research Agency (ANR).
Microcontroller (ULP-MCU) that allows decoding the address received in the WUB.

Ultra low power of WuRs is achieved at a cost of low sensitivity which limits their use in different applications that require a long range communication. WuR range has been evaluated showing the bottleneck of such technologies. In recent study [6] Basagni et al. evaluated the range of WuR in a real field deployment. The study shows that WuR can receive the quasi totality of sent packets at a distance up to $24 \mathrm{~m}$. Meanwhile, channel coding can be used to improve the sensitivity as presented by Rakovic et al. in [7]. The study compares the performance of Hamming code, repetition code and Walsh code applied to WuRs. It is shown that Walsh outperforms the other codes in reducing the packet error rate but at the cost of large addressing size (32B) and a high memory which is practically impossible in most of sensor nodes. Moreover, no experimental measurements were done.

Complex codes may provide good performance but induce an extra power consumption due to the complexity of decoding. Therefore, when considering applications for WSNs, only low complex codes would be conceivable. We propose in this work to take advantage of the computing capability of the WuR and add a simple channel coding to the WUB in order to enhance the WuR range. A promising channel coding, namely ME coding [8], is applied to WuRs in addition to Hamming coding. An energy model is proposed to compare the energy efficiency of both techniques and an evaluation of bit error probability and missed wake-ups is performed. Furthermore, experimental measurements in an anechoic chamber are presented, showing missed wake-ups reduction through channel coding. The experimentation was done in a real platform that was designed in [9].

This paper is structured as follows. Section II introduces WuR system design considered in this work and different channel coding schemes. Section III is devoted to the energy model before evaluations of bit error rate, false wake-up and energy consumption are presented in Section IV. Experimental measurements of missed wake-up are given in Section V. Finally Section VI concludes this paper.

\section{WAKE-UP RECEIVER DESIGN AND CHANNEL CODING}

\section{A. Wake-up receiver}

1) Architecture: WuR consists of an ultra low power receiver that is always listening to the channel while the main node is in sleep mode. Generally WuRs are based on an On-Off 
Keying (OOK) demodulator that transforms the radio signal into digital data bits that are decoded by an ULP-MCU or a correlator. The WuR considered in this work has been designed in [9]. It is based on a non-coherent receiver with an envelope detector and a hard decision with a comparator. The general blocks are illustrated in Fig. 1. The first block consists of a matching filter that allows a maximum transfer of power from the antenna to the receiver circuit at $868 \mathrm{MHz}$. Then, an envelope detector rectifies the signal to a baseband signal. This signal is compared to a threshold to reconstruct the bits of the WUB that passes through the ULP-MCU. A preamble detector serves to send an interrupt to the ULP-MCU, which is awaken from sleep state and can then decode the address embedded on the WUB. The ULP-MCU is a PIC12LF1552 microcontroller.

When the transmitter wants to send data to a destination, it first sends a WUB. The WUB contains a preamble and the address of the destination node. When using channel coding, the sequence of the address is mapped to a codeword according to the coding scheme. If no coding is used, the ULP-MCU of the WuR only checks the received address. However, when a channel coding is used, the ULP-MCU remaps the received codeword to the original symbol and then checks if the address is valid. Fig. 1 shows the required additional processing for channel decoding. If the received address is the good one, the $\mathrm{WuR}$ wakes-up the main receiver via an interrupt. The channel coding enhances the data transmission and thus a better detection of the WUB. Simple channel coding with low complexity are chosen in order to not penalize the power consumption and keeps the WuR consuming as low as possible with a better sensitivity.

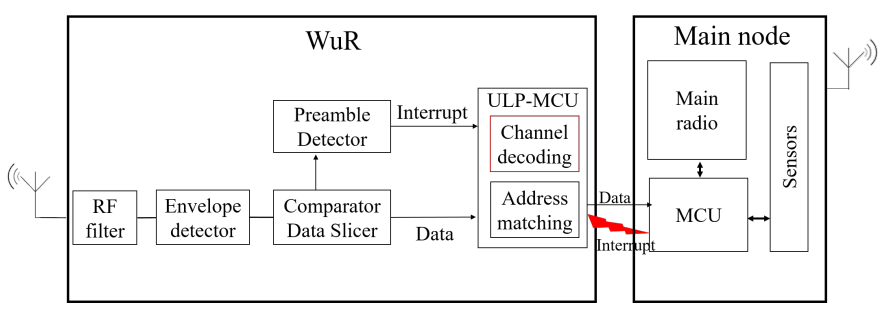

Fig. 1: WuR system design.

2) Bit error probability: The WuR sensitivity is directly linked to its bit error probability. Let $\mathrm{P}_{e S}$ and $\mathrm{P}_{e M}$ be the error probability of a low bit being received as a high bit and a high bit being received as a low bit, respectively.

For Non-Coherent OOK (NCOOK) receiver over an AWGN channel, $\mathrm{P}_{e S}$ and $\mathrm{P}_{e M}$ are expressed as [10]:

$$
P_{e S}=\exp \left(\frac{-b^{2}}{2 \sigma^{2}}\right)
$$

and

$$
P_{e M}=1-Q\left(\sqrt{\frac{2 E_{b}}{N_{0}}}, \frac{b}{\sigma}\right)
$$

with $E_{b}$ the received signal energy over 1-bit time interval, $\frac{N_{0}}{2}$ the power spectral density of the channel noise at the receiver input, $Q($.$) the Marcum- Q$ function.

$\sigma$ is the variance of additive white noise at the input of the envelope detector and $b$ is the decision threshold defined as:

$$
b=\sigma \sqrt{2+\frac{E_{b}}{2 N_{0}}} .
$$

The theoretical bit error probability $P_{e}$ of non-coherent receiver is:

$$
P_{e}=\frac{1}{2}\left(P_{e S}+P_{e M}\right) .
$$

A missed wake-up occurs when the WuR miss-detects the WUB and thus does not wake-up the main node while it should, inducing a missed wake-up. The missed wake-up probability denoted $P_{M W}$ is expressed as:

$$
P_{M W}=1-\left(1-P_{e}\right)^{k},
$$

with $k$ the size of the sent packet.

\section{B. Hamming coding}

1) Principle: In Hamming coding, a symbol is encoded by adding redundant bits. These redundant bits are inserted at certain positions in the symbol to perform error detection and correction process. The minimum distance of any code is given by $d_{m i n}$, and thus it can detect $d_{\min }-1$ bits and correct $\frac{d_{\min }-1}{2}$ bits. Hamming codes having a minimum distance $d_{\text {min }}=3$, can detect 2 error bits and correct 1 error bit.

2) Bit error probability: Considering a codeword of length $n$ and error correcting capability of $\frac{d_{\min }-1}{2}$ bits, and if the received codeword has a distance to the transmitted codeword greater than $\frac{d_{\min }-1}{2}$ then the probability of error denoted $P_{e}^{\text {Hamming }}$ is approximated as [11]:

$$
P_{e}^{\text {Hamming }} \approx \frac{1}{n}\left(2 \frac{d_{\min }-1}{2}+1\right) C_{n}^{\frac{d_{\min }-1}{2}+1} P_{e}^{\frac{d_{\min }-1}{2}+1} .
$$

\section{ME coding}

1) Principle: The idea of ME coding consists in mapping every $k$ bits of information into a $n$-bits codeword with at maximum one high bit as it can be shown in TABLE I. The all-zeros source symbol is mapped into a $n$-bits all-zeros codeword. All other source symbols are mapped into $n$-bits codewords with only one high bit in the $i^{t h}$ position of the source symbol. The codeword contains a total of $2^{k}-1$ bits.

Traditionally, when a received codeword contains more than one bit at '1', the processing unit of the receiver chooses a random symbol [8]. However, in our case the processing unit will not wait to receive the whole codeword that contains more than one bit at ' 1 ' to decode it. When the first one is received, it is directly mapped to its original symbol according to its position, then the processing unit will be turned off to save energy and reduce latency. When considering a transmitter 
TABLE I: Minimum energy coding mapping table.

\begin{tabular}{|c|c|}
\hline Source symbols $(k)$ & Mapped symbols $\left(n=2^{k}-1\right)$ \\
\hline \hline $00 . .00$ & $0000 . .000$ \\
$00 . .01$ & $0000 . .001$ \\
$\ldots$ & $\ldots$ \\
$\ldots$ & $\ldots$ \\
$\ldots$ & $\ldots$ \\
$11 . .10$ & $0100 . .000$ \\
$11 . .11$ & $1000 . .000$ \\
\hline
\end{tabular}

using OOK modulation that turns off the power amplifier when transmitting ' 0 ', the transmitter power consumption when applying ME coding is reduced as more ' 0 ' are sent than ' 1 '.

2) Bit error probability: When applying ME coding the probability of bit error denoted $P_{e}^{M E}$ is expressed as [8]:

$$
\begin{aligned}
P_{e}^{M E} & =\frac{1}{n+1}\left[\frac{n+1}{2} P_{e M}+\frac{n+3}{2} P_{e S}-P_{e M} P_{e S}\right. \\
& \left.-P_{e S}^{2}-\frac{1}{2}(n+1)\right]\left(1-P_{e S}\right)^{n-2}+\frac{1}{2} .
\end{aligned}
$$

\section{ENERGY MODEL}

To evaluate the energy optimization of both Hamming coding and ME coding schemes, the overall energy consumption including both transmitter and the WuR needs to be considered, as a node can be both transmitter and receiver in a WSN application. A point-to-point communication is considered with a source node and a destination node, where only the transmitter in the former node and the $\mathrm{WuR}$ in the later are switched on. The energy evaluation will be done for the targeted BER (also called bit error probability) required for all schemes. As ME coding reduces signal to noise ratio for the same BER, then the transmitted power can be reduced to achieve the same BER as uncoded scheme.

\section{A. Transmitter energy consumption}

The transmitter includes a modulator, a frequency synthesizer and a Power Amplifier (PA). The power consumption of the modulator and the frequency synthesizer can be considered as a constant $P_{c s t}$. The power consumption of the PA denoted $P_{P A}$ depends on the PA efficiency and the transmitted power $P_{\text {out }}$ [12]. $P_{\text {out }}$ depends on the channel conditions, the transmission distance, the carrier frequency, the targeted BER and the modulation scheme. It can be calculated as [11]:

$$
P_{\text {out }}=P_{L}(d) M R k_{B} T_{0} \frac{E_{b}}{N_{0}},
$$

with $R$ the bit rate, $\mathrm{M}$ the margin compensating the hardware process variations and other additive background noise including the noise figure, that is assumed to be equal to $100 \mathrm{~dB}$, which is a reasonable value for a WuR with a sensitivity around $-45 \mathrm{dBm}$. The average energy per noise spectral density $\frac{E_{b}}{N_{0}}$ depends on the targeted BER. The constant $k_{B} T_{0}$ is the thermal noise power spectral density at the temperature $T_{0}$ and $k_{B}$ the Boltzmann's constant. $P_{L}(d)$ is the path-loss expressed as [11]:

$$
P_{L}=\left(\frac{4 \pi d}{\lambda}\right)^{\gamma}
$$

with $\gamma$ the pathloss exponent that is equal to 2 in a free space, $\lambda$ the carrier wavelength that is equal to $\frac{c}{f}$, with $c$ the speed of light, and $f$ the carrier frequency. $d$ is the distance between the transmitter and the receiver.

The power consumption of the power amplifier can be approximated as [11]:

$$
P_{P A}=\frac{P_{\text {out }}}{\eta},
$$

with $\eta$ the drain efficiency of the power amplifier, its value is in the interval between 0 and 1 .

A circuit that turns off the PA when transmitting a ' 0 ' is considered, and thus the power consumption of transmitting ' 0 ' $P_{t x_{0}}$ is a low constant value, while the power consumption of transmitting ' 1 ' $P_{t x_{1}}$ depends on the power consumption of the PA and the other transmitter circuits $P_{c s t}$ and is expressed as [11]:

$$
P_{t x_{1}}=P_{P A}+P_{c s t} .
$$

The average transmitter power consumption is:

$$
P_{t x}=r_{d} P_{t x_{1}}+\left(1-r_{d}\right) P_{t x_{0}},
$$

with $r_{d}$ the high bit duty cycle (the amount of high bits $N_{H}$ transmitted over a packet of length $L_{b i t s}$ bits).

$$
r_{d}=\frac{N_{H}}{L_{b i t s}} .
$$

Considering a uniform distribution of ' 1 ' and ' 0 ', $r_{d}$ is equal to 0.5 with both uncoded scheme and with Hamming. However, when using $\operatorname{ME}(\mathrm{n}, \mathrm{k})$, there is $n$ bits at ' 1 ' over $n \times(n+1)$ bits of all codewords, and thus $r_{d}=\frac{1}{(n+1)}$.

Finally, the transmitter energy consumption with uncoded scheme per useful bit is:

$$
E_{t x}^{u n c o d e d}=\frac{P_{t x}}{R} .
$$

The transmitter energy consumption with either Hamming coding or ME coding per useful bit is:

$$
E_{t x}^{\text {Hamming }}=E_{t x}^{M E}=\frac{P_{t x}}{r R},
$$

with $\mathrm{r}$ the coding rate defined by $r=\frac{k}{n}$.

\section{B. WuR energy consumption}

Let $P_{r x}^{w u r}$ be the power consumption of the WuR when receiving a signal (i.e. when the ULP-MCU is turned on). When considering uncoded scheme, the energy consumption of the WuR per useful bit denoted $E_{\text {wur }}^{\text {uncoded }}$ is:

$$
E_{w u r}^{u n c o d e d}=\frac{P_{r x}^{w u r}}{R} .
$$


When applying Hamming $(\mathrm{n}, \mathrm{k})$, the energy consumption of the WuR per useful bit denoted $E_{\text {wur }}^{\text {Hamming }}$ is:

$$
E_{\text {wur }}^{\text {Hamming }}=\frac{P_{r x}^{\text {wur }}}{r R} .
$$

When applying $\operatorname{ME}(\mathrm{n}, \mathrm{k})$, as the codeword contains one bit at ' 1 ', the WuR turns off the ULP-MCU at the reception of the first ' 1 '. The average position of the bit at ' 1 ' denoted $L$ is equal to:

$$
L=\frac{n(n+3)}{2(n+1)} .
$$

The average energy consumption of the WuR per useful bit denoted $E_{\text {wur }}^{M E}$ is thus:

$$
E_{w u r}^{M E}=\frac{L P_{r x}^{w u r}}{k R} .
$$

\section{BER AND ENERGY EVALUATION}

In this section, the BER, the missed wake-up probability and the energy consumption are evaluated for uncoded scheme and when applying Hamming coding and ME coding to a noncoherent WuR.

\section{A. BER evaluation}

Fig. 2 shows the BER as a function of $\frac{E_{b}}{N_{0}}$ with uncoded scheme and when applying $\operatorname{ME}(7,3), \operatorname{ME}(15,4)$, Ham$\operatorname{ming}(7,4)$ and Hamming $(15,11)$. Both theoretical and montecarlo simulations are presented. Simulations were conducted in MATLAB. It can be seen that the simulation results fit the theoretical derivation.

A coding gain is defined as the reduction of the $\frac{E_{b}}{N_{0}}$ that is achieved at some specific level of BER when coding is used compared to the uncoded scheme. It can be seen that ME coding improves the BER compared to Hamming. With $\operatorname{ME}(7,3)$ and $\operatorname{ME}(15,4)$ at a BER of $10^{-3}, \frac{E_{b}}{N_{0}}$ is equal to $9.2 \mathrm{~dB}$ and $8.7 \mathrm{~dB}$, respectively and thus a coding gain of $1.8 \mathrm{~dB}$ and $2.3 \mathrm{~dB}$ is achieved. Meanwhile, with Hamming $(7,4)$ and Hamming $(15,11), \frac{E_{b}}{N_{0}}$ is equal to $10.7 \mathrm{~dB}$ and $10.1 \mathrm{~dB}$, respectively for the same level of BER, and thus a small coding gain of $0.3 \mathrm{~dB}$ and $0.9 \mathrm{~dB}$ is achieved. It can also be seen that at a fixed $\frac{E_{b}}{N_{0}}$, ME coding reduces the BER compared to the other schemes. Therefore, ME coding outperforms uncoded scheme and Hamming coding in either achieving better BER under the same energy consumption or achieving energy efficiency under the same BER.

\section{B. Missed wake-up evaluation}

When considering a WuR, it is interesting to evaluate the probability of missed wake-up. A missed wake-up occurs when an error arises on the received packet, and thus the WuR will not wake-up its main receiver, while it should. Fig. 3 shows the missed wake-up probability as a function of $\frac{E_{b}}{N_{0}}$ for all schemes. It can be seen that in case of uncoded scheme, the longer the size of the address is, the higher the probability to miss detect a packet is. However, when applying ME coding the more the size of the address is long, the less

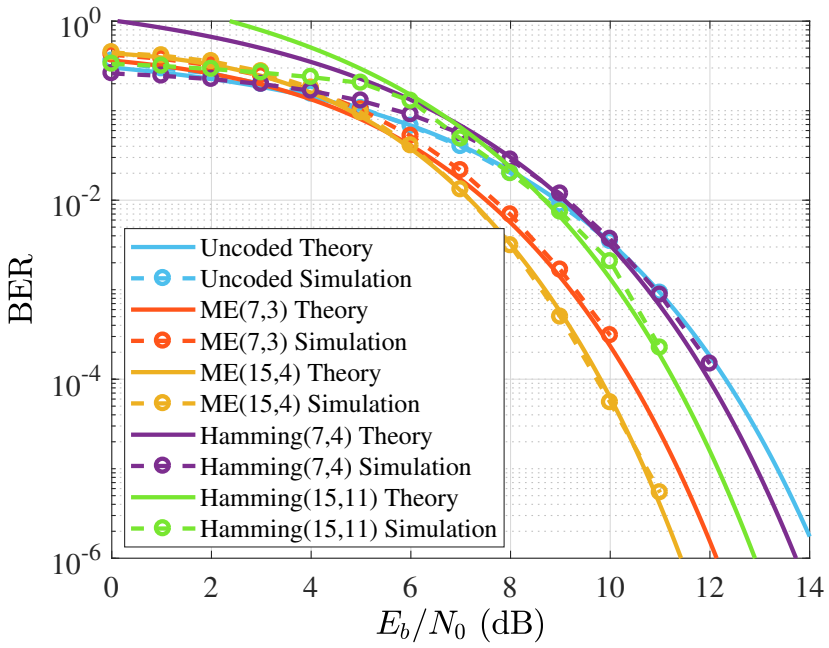

Fig. 2: BER as a function of $E_{b} / \mathrm{N}_{0}$.

the probability to miss a wake-up. ME coding outperforms Hamming coding and uncoded schemes in reducing the false wake-up probability, and thus the WuR is more susceptible to correctly detect the received address than the other schemes.

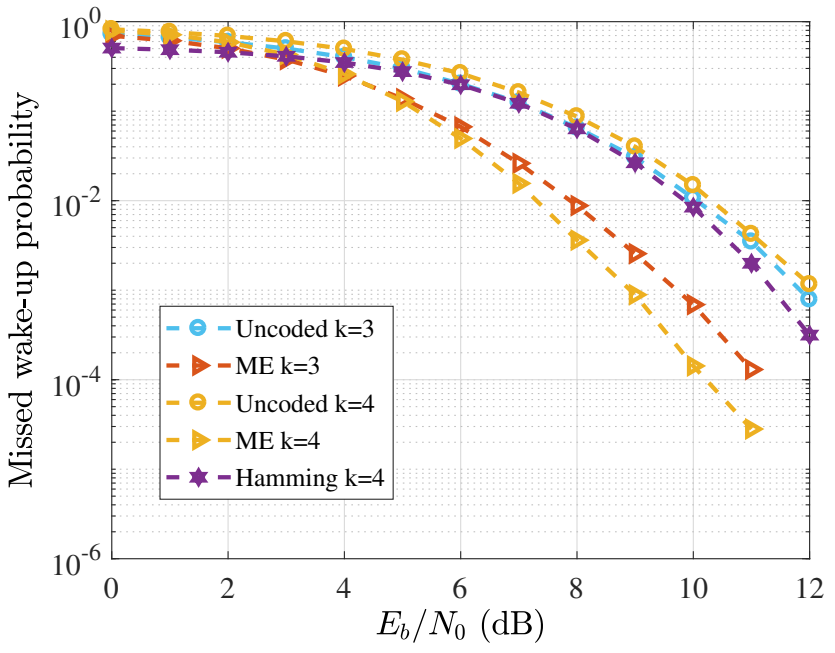

Fig. 3: Missed wake-up probability as a function of $E_{b} / N_{0}$.

\section{Energy consumption evaluation}

The ULP transmitter from STMicroelectronics is considered [13], from the datasheet it consumes $20 \mathrm{~mA}$ at a transmitted power of $14 \mathrm{dBm}$ and it can be deduced that $\eta=0.9$. For the receiver side, the WuR designed in [9] is considered, it consumes $284 \mu \mathrm{W}$ when receiving a signal and $1.83 \mu \mathrm{W}$ in idle listening.

The parameters used for the evaluation of the energy consumption are summarized in TABLE II to feed the energy model presented in Section III.

Fig. 4(a) shows the transmitter average energy consumption per useful bit at a bit error probability of $10^{-3}$ as a function of the range. It can be seen that for all schemes the transmitter 


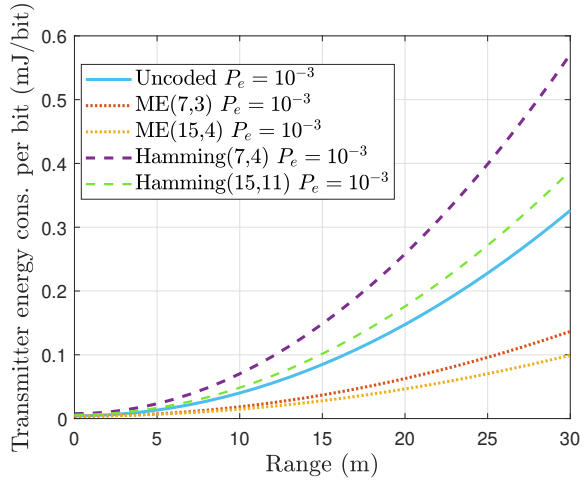

(a) Transmitter

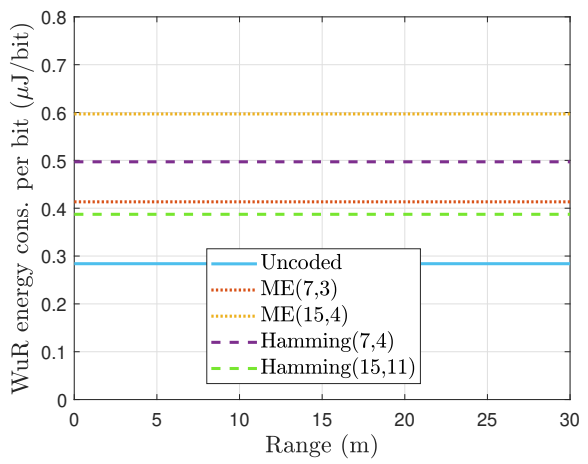

(b) $\mathrm{WuR}$

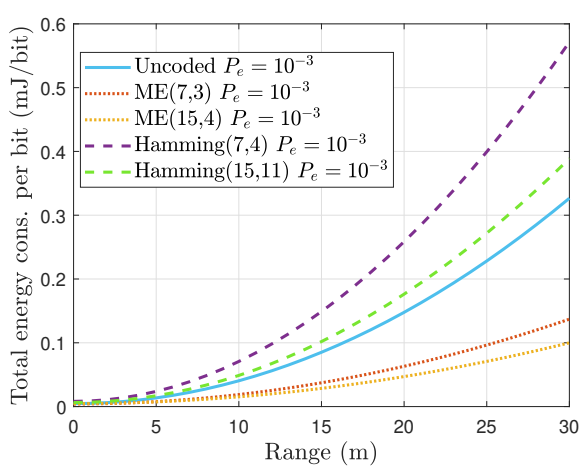

(c) Total

Fig. 4: Energy consumption per useful bit as a function of range.

TABLE II: Parameters used for the energy evaluation.

\begin{tabular}{|cc||cc|}
\hline Parameter & Value & Parameter & Value \\
\hline $\mathrm{f}$ & $868 \mathrm{MHz}$ & $\mathrm{M}$ & $100 \mathrm{~dB}$ \\
$T_{0}$ & $300 \mathrm{~K}$ & $k_{B}$ & $1.38 \cdot 10^{-23} \mathrm{Ws} / \mathrm{K}$ \\
$\mathrm{R}$ & $1 \mathrm{kbps}$ & $\eta$ & 0.9 \\
$P_{c s t}$ & $8.09 \mathrm{~mW}$ & $P_{t x_{0}}$ & $630 \mu \mathrm{W}$ \\
$P_{r x}^{\text {wur }}$ & $284 \mu \mathrm{W}$ & $P_{\text {idle }}^{\text {wur }}$ & $1.83 \mu \mathrm{W}$ \\
\hline
\end{tabular}

energy consumption per useful bit increases with the range, as a higher power transmission is required to achieve the required bit error probability at a longer range. ME coding achieves the lowest energy consumption per bit as the codeword contains more ' 0 ' than ' 1 ', and as the transmitter turns off the PA when transmitting ' 0 '. Furthermore, as ME coding has the best coding gain, it thus requires a lower power transmission than the other schemes. $\operatorname{ME}(7,3)$ and $\operatorname{ME}(15,4)$ consume, respectively 2.37 and 3.27 less than uncoded scheme at a distance of $28 \mathrm{~m}$. It can also be seen that when applying Hamming the energy consumption is higher than the other schemes, Hamming $(7,4)$ and Hamming $(15,11)$ consume, respectively 1.75 and 1.19 more than uncoded scheme as the codeword is longer. The transmitter is turned on for longer time and the small coding gain achieved with Hamming does not compensate the power consumption of the PA.

Fig. 4(b) shows the WuR average energy consumption per useful bit as a function of the range. It is clear that the energy consumption does not depend on the range. However it depends on the amount of time the ULP-MCU is on while decoding the received address. It can be seen that $\operatorname{ME}(15,4)$ consumes more than the other schemes as the code rate is higher so the WuR needs to decode more bits, letting the ULPMCU ON for a longer time.

Fig. 4(c) shows the total energy consumption per useful bit as a function of the range. It can be seen that when applying Hamming the total energy consumption is the highest. However, with $\operatorname{ME}(7,3)$ and $\operatorname{ME}(15,4)$ the total energy consumption is 2.38 and 3.25 , respectively less than uncoded scheme at a range of $28 \mathrm{~m}$. Thus the waste of energy consumption due to the address decoding with the WuR is compensated by the transmitter. As in a WSN application, a node can be both a

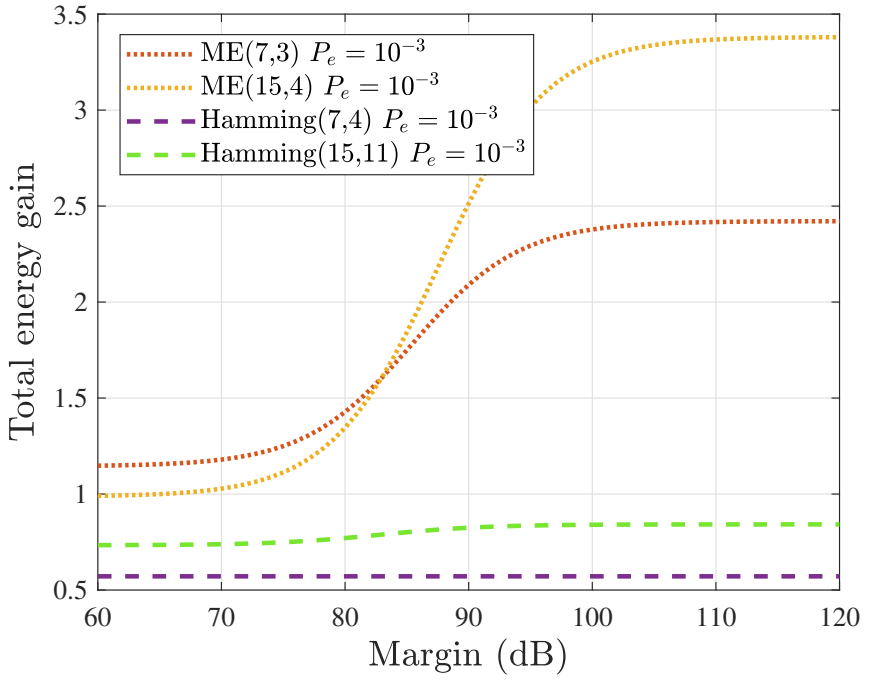

Fig. 5: Total energy gain at $d=28 \mathrm{~m}$ as a function of the margin.

receiver with a $\mathrm{WuR}$, and a transmitter, then the total energy consumption of a node can be reduced when applying ME coding.

Fig. 5 shows the total energy gain of each coding scheme compared to uncoded scheme at a distance of $28 \mathrm{~m}$ as a function of the margin. The energy gain is defined as the fraction between the total energy consumption when applying a coding over the total energy consumption with the uncoded scheme. The margin depends on the noise figure of the WuR, and thus the total energy gain is investigated for different circuits of WuRs that have different margin. It can be seen that with Hamming coding, a loss in the total energy consumption is achieved for all margin values. However, with ME coding a significant gain is achieved when the margin increases. With $\operatorname{ME}(7,3)$ and $\operatorname{ME}(15,4)$ a total energy gain up to 2.38 and 3.25 times, respectively is achieved with a margin equal to $100 \mathrm{~dB}$. This gain could be explained by the fact that with ME coding, to achieve the same bit error probability as uncoded scheme, a lower power transmission level is required compared 
to uncoded scheme thanks to the coding gain. Moreover, when the margin is high, the transmitter needs to transmit with higher power to achieve the required bit error probability at a fixed distance. As with ME coding more '0' are transmitted than ' 1 ', and when ' 0 ' is transmitted the PA is turned off, then the average power transmitted is lower than uncoded, resulting in a gain in the total energy consumption.

\section{EXPERIMENTAL MISSED WAKE-UP EVALUATION}

$\operatorname{ME}(7,3)$ has been implemented on the WuR designed in [9] and then the missed wake-up probability has been experimentally measured for 7215 packets sent at a rate of 4 packets/s. The missed wake-up probability represents the total wrongly received packets over the total transmitted packets. The WuR and the transmitter are placed at a distance of $1 \mathrm{~m}$ from each-other in an anechoic chamber as can be seen in Fig. 6. The missed wake-up probability is measured for different transmission power levels ranging from $-19.5 \mathrm{dBm}$ to $-16 \mathrm{dBm}$.

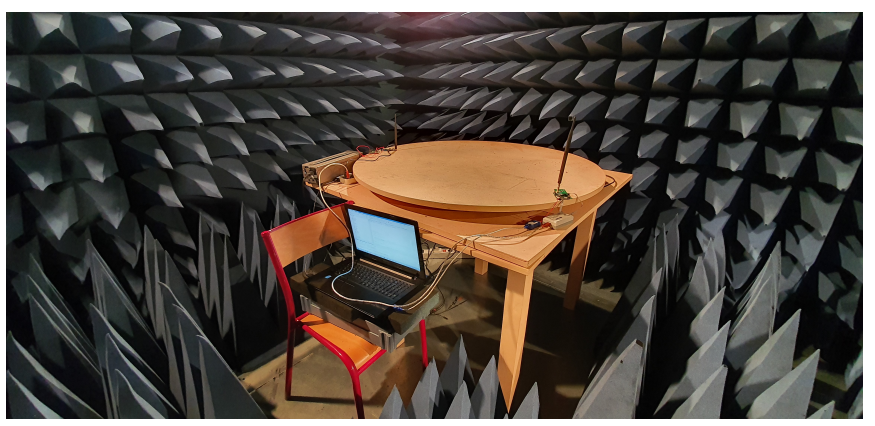

Fig. 6: Measurement environment.

Fig. 7 shows the measured missed wake-up probability as a function of the transmission power. It can be seen that under a transmission power higher than $-17 \mathrm{dBm}$ the missed wakeup probability is the same when applying $\operatorname{ME}(7,3)$ and with uncoded scheme. When the transmission power is lower than $17 \mathrm{dBm}$, it appears the gain of using $\operatorname{ME}(7,3)$ in either reducing the missed wake-up at the same transmission or reducing the transmission power at the same missed wake-up probability. At a transmission power of $-19 \mathrm{dBm}$, which is equivalent to a theoretical range of $28 \mathrm{~m}$ when using (7) and considering a transmission power of $10 \mathrm{dBm}$, the missed wake-up probability is improved up to $22 \%$ when applying $\operatorname{ME}(7,3)$.

\section{CONCLUSION}

This paper presented channel coding schemes that can be applied to WuRs with a detailed study of the probability of bit error rate, energy consumption and experimental measurements. Results show that Minimum Energy coding (ME) outperforms Hamming code and uncoded scheme in either reducing the bit error probability or saving energy. ME coding saves up to 3 times of energy compared to uncoded scheme at a range of $28 \mathrm{~m}$ and with a bit error probability of $10^{-3}$. To validate $\mathrm{ME}$ coding performance, experimental measurements have been conducted. $\operatorname{ME}(7,3)$ was implemented and the missed wake-up probability was measured in an anechoic chamber showing an improvement of $22 \%$ of the packets successfully received at a range of $28 \mathrm{~m}$.

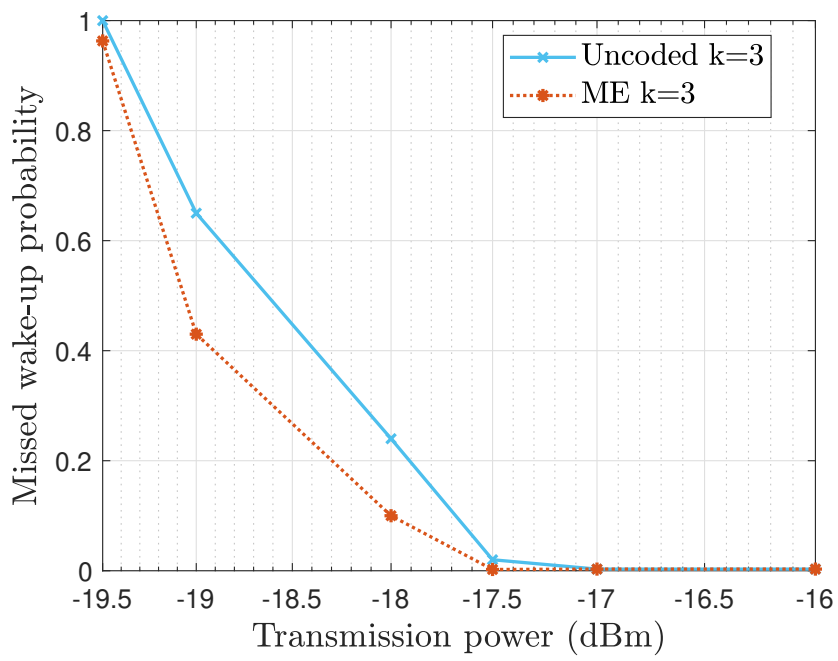

Fig. 7: Missed wake-up probability as a function of transmission power.

\section{REFERENCES}

[1] I. F. Akyildiz, W. Su, Y. Sankarasubramaniam, and E. Cayirci, "A survey on sensor networks," IEEE Communications Magazine, vol. 40, no. 8 , pp. 102-105, 2002.

[2] F. Ait Aoudia, M. Magno, M. Gautier, O. Berder, and L. Benini, "Analytical and Experimental Evaluation of Wake-Up Receivers Based Protocols," in IEEE Global Communications Conference, 2016, pp. 1-7.

[3] R. Piyare, A. L. Murphy, C. Kiraly, P. Tosato, and D. Brunelli, "Ultra Low Power Wake-Up Radios: A Hardware and Networking Survey," IEEE Communications Surveys \& Tutorials, vol. 19, no. 4, pp. $2117-$ 2157, 2017.

[4] J. Oller, I. Demirkol, J. Casademont, J. Paradells, G. U. Gamm, and L. Reindl, "Has Time Come to Switch from Duty-Cycled MAC Protocols to Wake-Up Radio for Wireless Sensor Networks?" IEEE/ACM Transactions on Networking, vol. 24, no. 2, pp. 674-687, 2016.

[5] N. E. H. Djidi, A. Courtay, M. Gautier, and O. Berder, "Adaptive relaying for wireless sensor networks leveraging wake-up receiver," in IEEE International Conference on Electronics, Circuits and Systems, 2018, pp. 797-800.

[6] S. Basagni, F. Ceccarelli, C. Petrioli, N. Raman, and A. V. Sheshashayee, "Wake-up radio ranges: A performance study," in IEEE Wireless Communications and Networking Conference, 2019, p. 6.

[7] V. Rakovic, R. Adamovski, A. Risteski, and L. Gavrilovska, "Improving Energy Efficiency and Reliability in WuR-Based IoT Systems: An Error Correction Approach," in Wireless Personal Communications, 2020.

[8] Q. Tang, S. Gupta, and L. Schwiebert, "BER performance analysis of an on-off keying based minimum energy coding for energy constrained wireless sensor applications," in IEEE International Conference on Communications (ICC), 2005, pp. 2734-2738.

[9] M. Magno, V. Jelicic, B. Srbinovski, V. Bilas, E. Popovici, and L. Benini, "Design, Implementation, and Performance Evaluation of a Flexible Low-Latency Nanowatt Wake-Up Radio Receiver,' IEEE Transactions on Industrial Informatics, vol. 12, no. 2, pp. 633-644, 2016.

[10] M. Schwartz, W. R. Bennett, and S. Stein, Communication systems and techniques. John Wiley \& Sons, 1995.

[11] Y. Peng, "Energy optimisation of communication techniques between communicating objects," Ph.D. dissertation, Université de Nantes, 2016.

[12] S. Cui, A. Goldsmith, and A. Bahai, "Modulation optimization under energy constraints," in IEEE International Conference on Communications (ICC), vol. 4, 2003, pp. 2805-2811.

[13] ST, Ultra-low power high performance sub-1 GHz transceiver Datasheet, 2019, accessed: 2020-05-19. [Online]. Available: https://www.st.com/content/st_com/en.html 